\title{
Observational constraints on dark matter-dark energy scattering cross section
}

\author{
Suresh Kumar ${ }^{1, a}$, Rafael C. Nunes ${ }^{2, b}$ \\ ${ }^{1}$ Department of Mathematics, BITS Pilani, Pilani Campus, Rajasthan 333031, India \\ 2 Departamento de Física, Universidade Federal de Juiz de Fora, Juiz de Fora, MG 36036-330, Brazil
}

Received: 4 September 2017 / Accepted: 25 October 2017 / Published online: 2 November 2017

(C) The Author(s) 2017. This article is an open access publication

\begin{abstract}
In this letter, we report precise and robust observational constraints on the dark matter-dark energy scattering cross section, using the latest data from cosmic microwave background (CMB) Planck temperature and polarization, baryon acoustic oscillations (BAO) measurements and weak gravitational lensing data from CanadaFrance-Hawaii Telescope Lensing Survey (CFHTLenS). The scattering scenario consists of a pure momentum exchange between the dark components, and we find $\sigma_{d}<$ $10^{-29} \mathrm{~cm}^{2}\left(m_{\mathrm{dm}} c^{2} / \mathrm{Gev}\right)$ at $95 \% \mathrm{CL}$ from the joint analysis $(\mathrm{CMB}+\mathrm{BAO}+\mathrm{CFHTLenS})$, where $m_{\mathrm{dm}}$ is a typical dark matter particle mass. We notice that the scattering among the dark components may influence the growth of large scale structure in the Universe, leaving the background cosmology unaltered.
\end{abstract}

\section{Introduction}

Cosmological observations reveal that approximately 95\% of the energy content of our Universe is unknown, and this matter/energy content is usually termed the dark sector of the Universe. Constraints from Planck team [1] show that $25 \%$ of this content is in the form of a non-relativistic matter called dark matter $(\mathrm{DM})$. The other part of this dark sector is in the form of an exotic component, dubbed dark energy (DE), with negative pressure responsible for accelerated expansion of the Universe at late times [2,3]. The particle physics experiments are yet to discover suitable particle candidates for the two dark components, and it is one of the greatest challenges in the contemporary physics. The popular candidate of $\mathrm{DE}$ is a positive cosmological constant [4-6] that suffers from theoretical inconsistencies, and consequently several alter-

\footnotetext{
${ }^{a}$ e-mail: suresh.kumar@pilani.bits-pilani.ac.in

be-mail: rafadcnunes@gmail.com
}

native models have been proposed in the literature to explain the late time accelerated expansion of the Universe [7].

Motivated to solve/assuage the theoretical problems in the $\Lambda \mathrm{CDM}$ model, models are proposed/studied in the literature where the dark components do not evolve separately but interact non-gravitationally with one another throughout the expansion of the Universe (see [8,9] for reviews). Essentially these interacting models invoke energy exchange between the dark components and a small degree of momentum exchange. These scenarios have been intensively investigated in the literature for several dark sector coupling functions and observational approximations [10-26]. It has recently been shown that the current observational data can favor the late time interaction between DE and DM [27-34].

Models with energy exchange modify the cosmology at background level (expansion history) and perturbative level (growth of structures). A scenario with a pure momentum exchange between the dark components is presented in [35], where the energy exchange between the dark components is negligible. Thus, the expansion history is the same as for a non-interacting scenario. This class of interaction considers an elastic scattering of the DM particles with DE. For, the DE presents a low density energy $\left(\sim 10^{-47} \mathrm{GeV}^{4}\right)$, and DM particles exhibit non-relativistic velocities dispersion, that is, DM is cold, or at least a considerable part of the DM is cold. Therefore, an elastic scattering appears as a natural modeling of the dark sector physics. On the other hand, elastic scattering is the most abundant process at the energy scales of interest. The model is independent of the microphysics involved in the scattering process, or the DE nature. Consequences of the elastic scattering interaction between the dark components on the growth of large scale structure are presented in [35], while its effects on linear and nonlinear structure formation via $N$-body simulations are investigated in $[36,37]$. In the next section, we present more details as regards this interaction model. 
The aim of this paper is to constrain the DM-DE scattering cross section (as well as the equation of state parameter of $\mathrm{DE}$ in this context) with precision and robustness using the latest observational data from CMB Planck temperature and polarization, BAO measurements and CFHTLenS data. In next section, we introduce the model with the elastic scattering between DM and DE. We present the results and the related discussion of our analysis in Sect. 3. In the final section, we summarize the findings of our study with future perspectives.

\section{Dark matter-dark energy scattering models}

The interaction between particles can be quantified by the cross section (the likelihood within an area transverse to their relative motions) within which the particles must meet in order to scatter from each other, implying a transfer of momentum and/or energy, or lead to the creation of new particles. At low energies the cross section can be well described by a process of elastic scattering. For instance, the photon-baryons fluid (essentially photon-electrons) in the early Universe is given in terms of the Thomson cross section, $\sigma_{T} \simeq 10^{-25} \mathrm{~cm}^{2}$. In general, the drag force can be written as

$\mathbf{F}=-(1+w) \sigma \gamma^{2} \rho \mathbf{v}$

where $v$ is the velocity of the particle traversing the fluid, and $\gamma$ is the Lorentz factor. For instance, the photon-electron coupling $(w=1 / 3)$ is given by $\mathbf{F}=-4 / 3 \sigma_{T} v \rho_{\gamma}$.

Considering the drag force 1 as the only non-gravitational force between DE and DM, the linear perturbation theory equations (in conformal newtonian gauge) for DE-DM interaction fluids are given by $[35,38]$

$$
\begin{aligned}
\theta_{\mathrm{de}}^{\prime}= & 2 \mathcal{H} \theta_{\mathrm{de}}+k^{2} \frac{\delta_{\mathrm{de}}}{1+w_{\mathrm{de}}}+k^{2} \Psi \\
& -a n_{\mathrm{dm}} \sigma_{d}\left(\theta_{\mathrm{de}}-\theta_{\mathrm{dm}}\right)
\end{aligned}
$$

and

$$
\begin{aligned}
\theta_{\mathrm{dm}}^{\prime}= & -\mathcal{H} \theta_{\mathrm{dm}}+k^{2} \Psi \\
& +\frac{\rho_{\mathrm{de}}}{\rho_{\mathrm{dm}}}\left(1+w_{\mathrm{de}}\right) a n_{\mathrm{dm}} \sigma_{d}\left(\theta_{\mathrm{de}}-\theta_{\mathrm{dm}}\right),
\end{aligned}
$$

where $n_{\mathrm{dm}}$ is the proper number density of DM particles, $\sigma_{d}$ is the scattering cross section between DE and DM.

The velocity perturbation above exhibits a new drag term,

$S=a n_{\mathrm{dm}} \sigma_{d}\left(\theta_{\mathrm{de}}-\theta_{\mathrm{dm}}\right)$

It represents the DE fraction which is subject to scattering per unit time. In this scenario, the equation of continuity, $\delta_{i=\mathrm{dm}, \mathrm{de}}=\delta \rho_{i=\mathrm{dm}, \mathrm{de}} / \rho_{i=\mathrm{dm}, \mathrm{de}}$, remains unchanged and follows the standard evolution. As already commented above, this scenario assumes that the dark components are not coupled at background level. Here, the interaction in the dark sector is quantified by the drag term $S$, and only acts via the velocity perturbation equations.

Once that $\rho_{\mathrm{dm}}=m_{\mathrm{dm}} n_{\mathrm{dm}}$ for DM particles, where $m_{\mathrm{dm}}$ stands for the mass of a typical DM particle, then Eq. (4) can be rewritten as

$S=a \rho_{\mathrm{dm}} \xi\left(\theta_{\mathrm{de}}-\theta_{\mathrm{dm}}\right)$,

where we have defined

$\xi=\frac{\sigma_{d}}{m_{\mathrm{dm}}}$,

with dimensions/units of barn $\cdot c^{2} / \mathrm{GeV}$, as the characteristic parameter of the drag term.

\section{Results}

In order to constrain the cross section of elastic scattering between DE and DM, we consider the following data sets (briefly described) sensitive at the perturbations level.

$C M B$ We use the full Planck 2015 data [1] comprised of temperature (TT), polarization (EE) and the cross correlation of temperature and polarization (TE) together with the CMB lensing power spectrum.

$B A O$ We use the BAO measurements from the Six Degree Field Galaxy Survey (6dF) [39], the Main Galaxy Sample of Data Release 7 of Sloan Digital Sky Survey (SDSSMGS) [40], the LOWZ and CMASS galaxy samples of the Baryon Oscillation Spectroscopic Survey (BOSS-LOWZ and BOSS-CMASS, respectively) [41], and the distribution of the LymanForest in BOSS (BOSS-Ly) [42]. These data points are summarized in Table I of [43].

CFHTLenS We consider the full likelihood of the weak gravitational lensing data from the blue galaxy sample compiled in [44].

The base parameters set for the DM-DE scattering model, to be constrained, is given by

$P=\left\{100 \omega_{\mathrm{b}}, \omega_{\mathrm{dm}}, 100 \theta_{s}, \ln 10^{10} A_{s}, n_{s}, \tau_{\text {reio }}, \xi, w_{\mathrm{de}}\right\}$,

where the first six parameters are the base parameters for the standard $\triangle \mathrm{CDM}$ model (see [1] for more details) while the remaining two parameters correspond to its extension in the present study.

For DE properties, we assume that its equation of state parameter is constant ( $w_{\mathrm{de}}=$ constant $)$ and we have taken 
Table 1 Constraints on the free parameters and some derived parameters of the DM-DE scattering model. Mean values of the parameters are displayed with $1 \sigma$ and $2 \sigma$ CL except the parameter $\xi$ for which the $2 \sigma$ upper bound is mentioned. The parameter $H_{0}$ is in the units of $\mathrm{km} \mathrm{s}^{-1} \mathrm{Mpc}^{-1}$. Final row carries the $\chi_{\min }^{2} / 2$ values for the three cases of data-fitting

\begin{tabular}{llll}
\hline Parameter & $\mathrm{CMB}$ & $\mathrm{CMB}+\mathrm{BAO}$ & $\mathrm{CMB}+\mathrm{BAO}+\mathrm{CFHTLenS}$ \\
\hline $100 \omega_{\mathrm{b}}$ & $2.227_{-0.014-0.026}^{+0.014+0.027}$ & $2.231_{-0.016-0.028}^{+0.014+0.030}$ & $2.238_{-0.013-0.025}^{+0.013+0.026}$ \\
$\omega_{\mathrm{dm}}$ & $0.1193_{-0.0015-0.0024}^{+0.0013+0.0026}$ & $0.1186_{-0.0013-0.0024}^{+0.0013+0.0024}$ & $0.1179_{-0.0011-0.0024}^{+0.0012+0.0023}$ \\
$100 \theta_{s}$ & $1.04189_{-0.00029-0.00057}^{+0.00029+0.00056}$ & $1.04195_{-0.00028-0.00055}^{+0.00028+0.00055}$ & $1.04191_{-0.00031-0.000058}^{+0.00027+0.00060}$ \\
$\ln 10^{10} A_{s}$ & $3.060_{-0.024-0.041}^{+0.020+0.043}$ & $3.069_{-0.027-0.043}^{+0.023+0.048}$ & $3.061_{-0.027-0.043}^{+0.022+0.047}$ \\
$n_{s}$ & $0.9649_{-0.0042-0.0079}^{+0.0042+0.0080}$ & $0.9667_{-0.0048-0.0096}^{+0.0048+0.0095}$ & $0.9683_{-0.0045-0.0086}^{+0.0045+0.0091}$ \\
$\tau_{\text {reio }}$ & $0.064_{-0.013-0.022}^{+0.011+0.022}$ & $0.069_{-0.015-0.025}^{+0.013+0.027}$ & $0.066_{-0.015-0.024}^{+0.012+0.026}$ \\
$\xi$ & $<9.8 \times 10^{-5}$ & $<9.8 \times 10^{-5}$ & $<9.4 \times 10^{-5}$ \\
$w_{\mathrm{de}}$ & $-1.11_{-0.25-0.30}^{+0.14+0.37}$ & $-1.06_{-0.06-0.13}^{+0.07+0.12}$ & $-1.03_{-0.06-0.12}^{+0.06+0.11}$ \\
$H_{0}$ & $71.2_{-5.1-10}^{+7.7+10}$ & $69.6_{-1.8-3.2}^{+1.5+3.4}$ & $68.9_{-1.6-2.9}^{+1.4+3.0}$ \\
$\sigma_{8}$ & $0.848_{-0.044-0.100}^{+0.067+0.089}$ & $0.834_{-0.018-0.036}^{+0.018+0.039}$ & $0.819_{-0.016-0.030}^{+0.016+0.032}$ \\
$\chi_{\min }^{2} / 2$ & 6475.61 & 6481.87 & 6532.69 \\
\hline
\end{tabular}

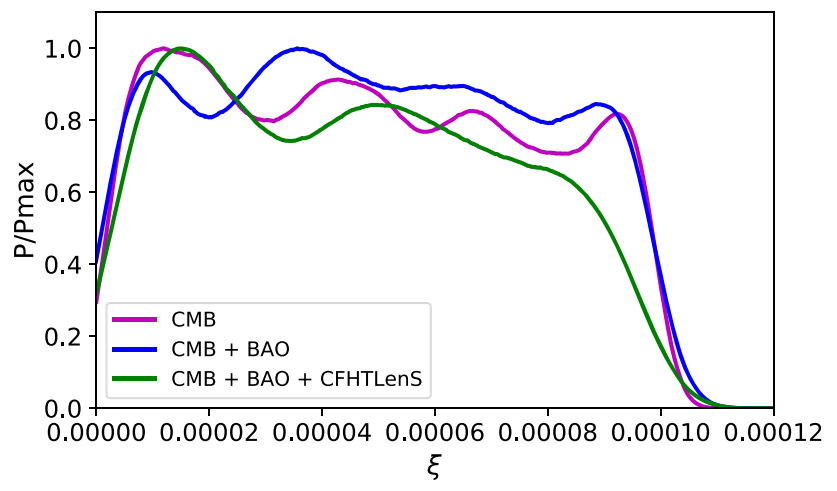

Fig. 1 One-dimensional marginalized probability distribution of $\xi$

$c_{s}^{2}=\delta p_{\mathrm{de}} / \delta \rho_{\mathrm{de}}=1\left(c_{s}^{2}\right.$ is in units of the speed of light $)$. This necessarily implies that DE is a very light scalar field, as a typical canonical scalar field model with mass $\lesssim H_{0}$. Also, in order to avoid the unphysical sound speed, usually we take $c_{s}^{2}=1$. We consider suitable uniform priors on the parameters of the model under consideration. In particular, we choose $\xi \in\left[10^{-9}, 10^{-3}\right]$. We take $10^{-3}$ as an upper bound, since values greater than that return surreal values to $\sigma_{8}$. On the other hand, it is reasonable to expect that $\xi<<1$.

We modified the publicly available CLASS [45] and Monte Python [46] codes for the DM-DE scattering model under consideration, and constrained the model parameters by utilizing three different combinations of data sets: $\mathrm{CMB}$ alone, $\mathrm{CMB}+\mathrm{BAO}$ and $\mathrm{CMB}+\mathrm{BAO}+\mathrm{CFHTLenS}$. We used Metropolis Hastings algorithm on the model parameters to obtain correlated Markov Chain Monte Carlo (MCMC) samples from CLASS/Monte Python code, and finally analyzed these samples by using the GetDist Python package [47].

Table 1 summarizes the main results of the statistical analysis carried out using three different combinations of data sets: $\mathrm{CMB}$ alone, $\mathrm{CMB}+\mathrm{BAO}$ and $\mathrm{CMB}+\mathrm{BAO}+$ CFHTLenS. The one-dimensional marginalized distribution of $\xi$ is shown in Fig. 1 while one-dimensional marginalized distribution, two-dimensional $1 \sigma$ and $2 \sigma$ confidence contours for some selected parameters are displayed in Fig. 2.

In all the three cases, we note that $\xi \lesssim 10^{-4}$. From Eq. (6), we can write the cross section between DM and DE as

$\sigma_{d}<10^{-29} \mathrm{~cm}^{2}\left(m_{\mathrm{dm}} \frac{c^{2}}{\mathrm{Gev}}\right)$

or in terms of the Thomson cross section value, $\sigma_{T} \simeq 10^{-25}$ $\mathrm{cm}^{2}$, as

$\sigma_{d}<10^{-4} \sigma_{T} \mathrm{~cm}^{2}\left(m_{\mathrm{dm}} \frac{c^{2}}{\mathrm{Gev}}\right)$.

The typical DM particle mass (as weakly interacting massive particle (WIMP) candidate) can be taken in the range $1-1000 \mathrm{GeV} / c^{2}$. Recently, the best upper limits for WIMP masses are found as $10 \mathrm{GeV} / c^{2}, 40 \mathrm{GeV} / c^{2}$, and $50 \mathrm{GeV} / c^{2}$ from the experiments XENON1T [48], PandaX-II [49], and LUX [50], respectively. In order to qualitatively discuss our results, taking $m_{\mathrm{dm}}=10 \mathrm{GeV} / c^{2}$, we expect to have $\sigma_{d} \lesssim 10^{-3} \sigma_{T} \mathrm{~cm}^{2}$ at $95 \% \mathrm{CL}$. As expected, we can note that the interaction (non-gravitational) between the dark components is too small, at least three orders of magnitude lower than the photon-electron interaction.

The constraints obtained by using CMB data alone show a small preference for a phantom dynamics, i.e., $w_{\text {de }} \lesssim-1$. Such a constraint on $w_{\text {de }}$ from CMB has also been observed in [51-53]. In the present study, we may infer that the DM particles undergo elastic scattering with scalar fields having negative kinetic term. It is well known that these fields suffer from instabilities at the classical and quantum levels [54,55] that casts doubts about their existence. Nevertheless, observationally such fields cannot be discarded, as we have also observed here. 


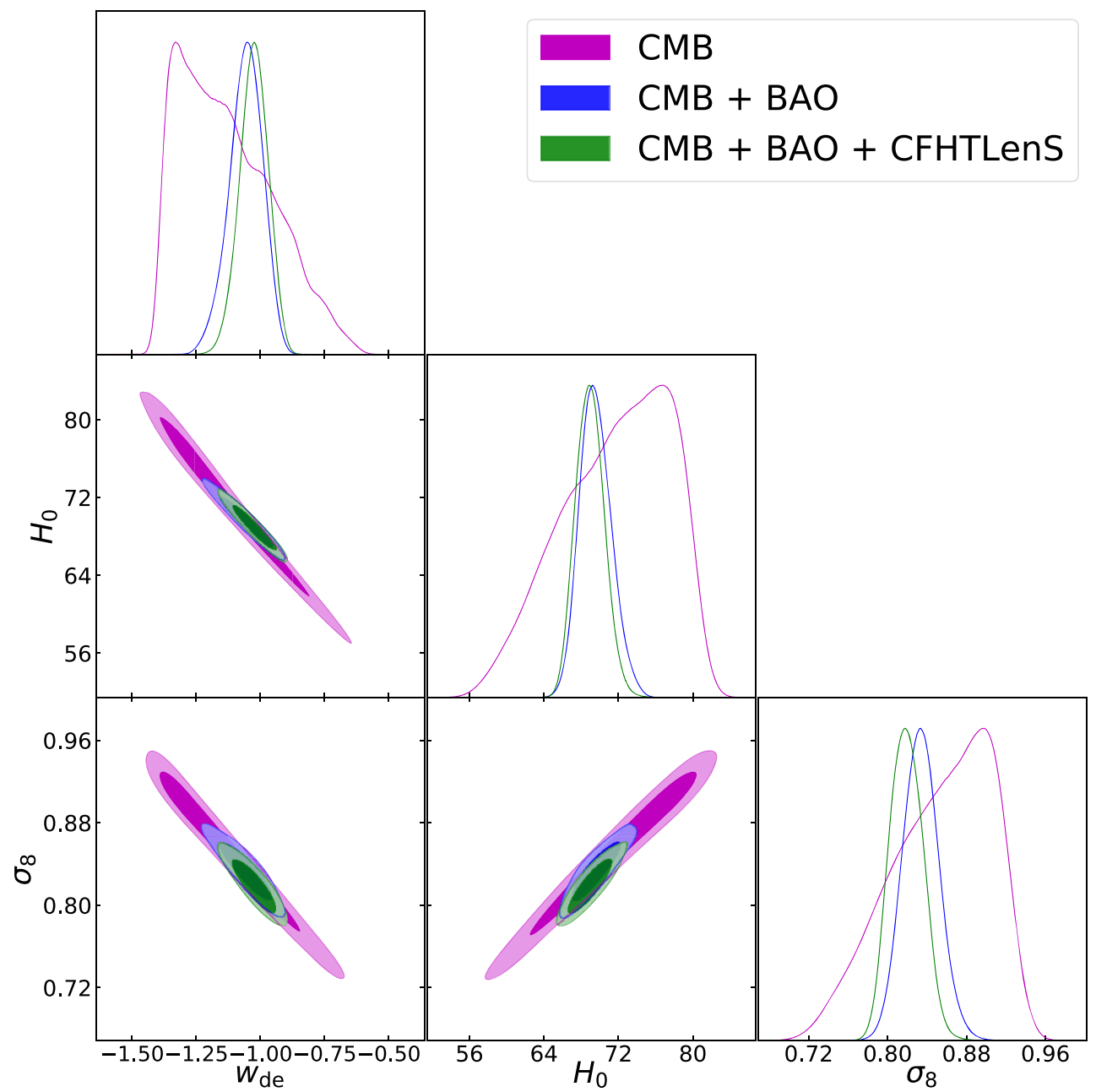

Fig. 2 One-dimensional marginalized distribution, two-dimensional $1 \sigma$ and $2 \sigma$ confidence contours for some selected parameters

From Table 1 and Fig. 2, it is clear that the combined data set $\mathrm{CMB}+\mathrm{BAO}+\mathrm{CFHTLenS}$ yields the tightest constraints on the model parameters. In order to observe/show the quantitative effects of $\xi$ on the power spectrum $P(k)$ of matter at $z=0$, and CMB TT power spectrum in contrast with the base line Planck $2015 \Lambda$ CDM model [1], we select some particular values of $\xi$ from its $95 \%$ confidence region given by the combined data set $\mathrm{CMB}+\mathrm{BAO}+\mathrm{CFHTLenS}$. Figures 3 and 4 depict the relative deviation of CMB TT and matter power

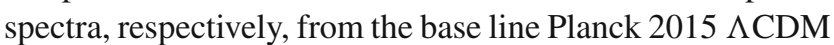
model (red line) for the three values of $\xi$ as mentioned in the legends of the figures while the other parameters are fixed to their best-fit mean values as given in Table 1 . We see that relative deviation of CMB TT power spectrum varies from the percent level to $8 \%$. On the other hand, significant relative deviation of matter power spectrum can be observed for the different values of $\xi$ in Fig. 4. It is clear that the larger values of $\xi$ tend to cause significant effects on the two power spectra.



Fig. 3 Relative deviation of CMB TT power spectrum from the base line Planck $2015 \Lambda \mathrm{CDM}$ model (red line) for various values of $\xi$ while the other parameters are fixed to their best-fit mean values as given in Table 1 


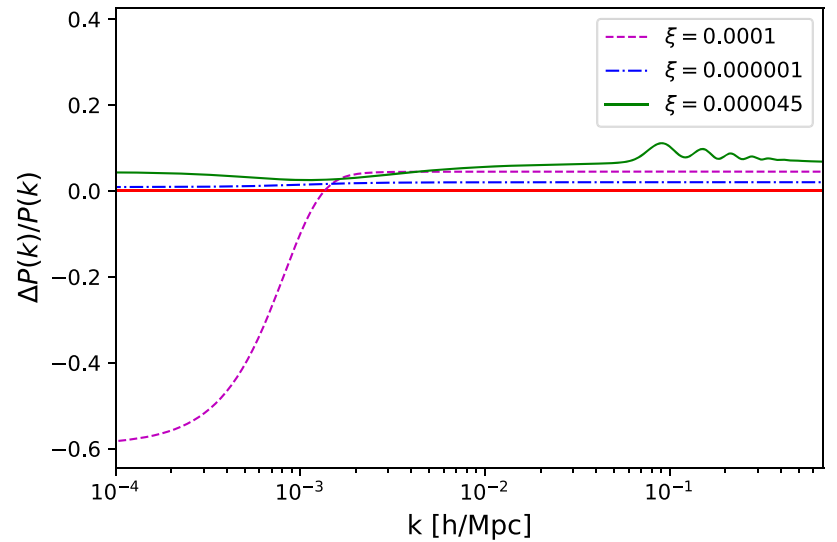

Fig. 4 Relative deviation of matter power spectrum from the base line Planck $2015 \Lambda \mathrm{CDM}$ model (red line) for various values of $\xi$ while the other parameters are fixed to their best-fit mean values as given in Table 1

\section{Conclusion and perspectives}

In this study, we have considered an elastic scattering between DM and DE to constrain the cross section between the dark components. This elastic scattering for DM-DE is analogous to the Thomson scattering for baryons and photons. We find $\sigma_{d} \lesssim 10^{-28} \mathrm{~cm}^{2}$ at $95 \% \mathrm{CL}$ from our joint analysis (CMB + BAO + CFHTLenS), for typical DM mass of the order $10 \mathrm{GeV} / c^{2}$. This quantifies a very small interaction among the dark components. We find that the combined data set $\mathrm{CMB}+\mathrm{BAO}+\mathrm{CFHTLenS}$ puts the tightest constraints on the model parameters when compared with the other two cases considered in this study, and some possible values of $\xi$ can cause significant changes in the matter power spectrum $P(k)$.

It may be noted that the DM-DE scattering model studied here reduces to the standard $\Lambda \mathrm{CDM}$ model with $\xi=0$ and $w_{\mathrm{de}}=-1$. The constraints in Table 1 for the combined data set do not differ considerably from the ones obtained in [1] for the base line Planck $2015 \Lambda \mathrm{CDM}$ model. Therefore, the presence of the non-zero parameter $\xi$ does not yield significant changes in the background cosmological dynamics. However, as pointed out in [35], the elastic scattering among dark components may influence the growth of large scale structure in the Universe (see Figs. 3 and 4). The precise and robust constraints obtained in the present study may be utilized for studying the linear and nonlinear structure formation in the DM-DE elastic scattering model via $N$-body simulations (see $[36,37])$. On the other hand, one can generalize the DM-DE elastic scattering scenario investigated here by considering an energy exchange between DM and DE, beyond the pure momentum exchange, that is, a modified model with cosmological effects at background level (expansion history) and perturbative level (growth of structures) using a general parametrization of DE coupled to DM. Also, it could be worthwhile to investigate an elastic scattering between DE and massive neutrinos (and/or dark radiation). Progress in this direction will be reported in a forthcoming paper.

Acknowledgements The authors are grateful to Bharat Ratra for constructive and fruitful discussions. SK gratefully acknowledges the support from SERB-DST project No. EMR/2016/000258.

Open Access This article is distributed under the terms of the Creative Commons Attribution 4.0 International License (http://creativecomm ons.org/licenses/by/4.0/), which permits unrestricted use, distribution, and reproduction in any medium, provided you give appropriate credit to the original author(s) and the source, provide a link to the Creative Commons license, and indicate if changes were made.

Funded by $\mathrm{SCOAP}^{3}$.

\section{References}

1. P.A.R. Ade et al., A\&A 594, A13 (2016). Planck collaboration, arXiv: 1502.01589

2. A.G. Reiss et al., Astron. J. 116, 1009 (1998). arXiv:astro-ph/9805201

3. S.J. Perlmutter et al., Astrophys. J. 517, 565 (1999). arXiv:astro-ph/9812133

4. S. Weinberg, Rev. Mod. Phys. 61, 1 (1989)

5. T. Padamanbhan, Phys. Rep. 380, 235 (2003). arXiv:hep-th/0212290

6. R. Bousso, Gen. Relativ. Gravit. 40, 607 (2008). arXiv:0708.4231

7. E.J. Copeland, M. Sami, S. Tsujikawa, Int. J. Mod. Phys. D 15, 1753 (2006). arXiv:hep-th/0603057

8. A. YuL Bolotin, O.A. Kostenko, D.A.Yerokhin Lemets, Int. J. Mod. Phys. D 24, 1530007 (2015). arXiv:1310.0085

9. B. Wang, E. Abdalla, F. Atrio-Barandela, D. Pavón, Rep. Prog. Phys. 79, 096901 (2016). arXiv:1603.08299

10. W. Yang, N. Banerjee, S. Pan, Phys. Rev. D 95(12), 123527 (2017). arXiv: 1705.09278

11. F.E.M. Costa, E.M. Barboza Jr., J.S. Alcaniz, Phys. Rev. D 79, 127302 (2009). arXiv:0905.0672

12. R.C. Nunes, E.M. Barboza Jr., Gen. Relativ. Gravit. 46, 1820 (2014). arXiv: 1404.1620

13. R.C. Nunes, S. Pan, E.N. Saridakis, Phys. Rev. D 94, 023508 (2016). arXiv: 1605.01712

14. A. Pourtsidou, T. Tram, Phys. Rev. D 94, 043518 (2016). arXiv: 1604.04222

15. W. Yang, H. Li, Y. Wu, J. Lu, JCAP 10, 007 (2016). arXiv: 1608.07039

16. R.Y. Guo, Y.H. Li, J.F. Zhang, X. Zhang, JCAP 05, 040 (2017). arXiv: 1702.04189

17. N. Tamanini, Phys. Rev. D 92, 043524 (2015). arXiv:1504.07397

18. G.S. Sharov et al., Mon. Not. R. Astron. Soc. 466 (2017). arXiv: 1701.00780

19. Y. Wang, D. Wands, L. Xu, J.D. Santiago, A. Hojjati, Phys. Rev. D 87, 083503 (2013). arXiv:1301.5315

20. R.J.F. Marcondes, R.C.G. Landim, A.A. Costa, B. Wang, E. Abdalla, JCAP 12, 009 (2016). arXiv:1605.05264

21. C. van de Bruck, J. Mifsud, J.P. Mimoso, N.J. Nunes, JCAP 1611(11), 031 (2016). arXiv: 1605.03834

22. J. Valiviita, E. Palmgren, JCAP 1507, 015 (2015). arXiv: 1504.02464

23. D. Duniya, D. Bertacca, R. Maartens, Phys. Rev. D 91, 063530 (2015). arXiv: 1502.06424

24. S. Pan, A. Mukherjee, N. Banerjee, arXiv:1710.03725 [astroph.CO] 
25. J.J. Guo, J.F. Zhang, Y.H. Li, D.Z. He, X. Zhang, arXiv:1710.03068 [astro-ph.CO]

26. X. Xu, Y.Z. Ma, A. Weltman, arXiv:1710.03643 [astro-ph.CO]

27. V. Salvatelli, N. Said, M. Bruni, A. Melchiorri, D. Wands, Phys. Rev. Lett. 113(18), 181301 (2014). arXiv:1406.7297

28. J. Solà, A. Gomez-Valent, J. de Cruz, Prez. Astrophys. J. 811, L14 (2015). arXiv: 1506.05793

29. R. Murgia, S. Gariazzo, N. Fornengo, JCAP 1604(04), 014 (2016). arXiv: 1602.01765

30. C. Pigozzo, S. Carneiro, J.S. Alcaniz, H.A. Borges, J.C. Fabris, JCAP 1605, 022 (2016). arXiv: 1510.01794

31. S. Kumar, R.C. Nunes, Phys. Rev. D 94, 123511 (2016). arXiv: 1608.02454

32. S. Kumar, R.C. Nunes, arXiv: 1702.02143

33. E.D. Valentino, A. Melchiorri, O. Mena, Phys. Rev. D 96, 043503 (2017). arXiv: 1704.08342

34. E.G.M. Ferreira, J. Quintin, A.A. Costa, E. Abdalla, B. Wang, Phys. Rev. D 95, 043520 (2017). arXiv: 1412.2777

35. F. Simpson, Phys. Rev. D 82, 083505 (2010). arXiv:1007.1034

36. M. Baldi, F. Simpson, Mon. Not. R. Astron. Soc. 465, 1 (2017). arXiv:1605.05623

37. M. Baldi, F. Simpson, Mon. Not. R. Astron. Soc. 449, 3 (2015). arXiv: 1412.1080

38. C.P. Ma, E. Bertschinger, Astrophys. J. 7, 455 (1995). arXiv:astro-ph/9506072

39. F. Beutler, C. Blake, M. Colless, D.H. Jones, L. Staveley-Smith, L. Campbell, Q. Parker, W. Saunders, F. Watson, MNRAS 416, 3017 (2011). arXiv:1106.3366
40. A.J. Ross, L. Samushia, C. Howlett, W.J. Percival, A. Burden, M. Manera, MNRAS 449, 835 (2015). arXiv: 1409.3242

41. L. Anderson et al., MNRAS 441, 24 (2014). arXiv: 1312.4877

42. A. Font-Ribera et al., JCAP 5, 27 (2014). arXiv:1311.1767

43. R.C. Nunes, S. Pan, E.N. Saridakis, E.M.C. Abreu, JCAP 1701(01), 005 (2017). arXiv: 1610.07518

44. C. Heymans et al., Mon. Not. R. Astron. Soc. 432, 2433 (2013). arXiv: 1303.1808

45. D. Blas, J. Lesgourgues, T. Tram, JCAP 07, 034 (2011). arXiv: 1104.2933

46. B. Audren, J. Lesgourgues, K. Benabed, S. Prunet, JCAP 02, 001 (2013). arXiv: 1210.7183

47. https://github.com/cmbant/getdist

48. E. Aprile et al., arXiv:1705.06655 [astro-ph.CO]

49. Andi Tan et al., Phys. Rev. Lett. 117, 121303 (2016). arXiv: 1607.07400

50. D.S. Akerib et al., Phys. Rev. Lett. 118, 021303 (2017). arXiv:1608.07648

51. E. Di Valentino, A. Melchiorri, J. Silk, Phys. Lett. B 761 (2016). arXiv:1606.00634

52. D.L. Shaferm, D. Huterer, Phys. Rev. D 89, 063510 (2014). arXiv: 1312.1688

53. Cheng Cheng, Qing-Guo Huang, Phys. Rev. D 89, 043003 (2014). arXiv: 1306.4091

54. F. Sbisa, Eur. J. Phys. 36, 015009 (2015). arXiv:1406.4550

55. S.M. Carroll, M. Hoffman, M. Trodden, Phys. Rev. D 68, 023509 (2003) 